\title{
Féeries
}

Études sur le conte merveilleux, XVII ${ }^{\mathrm{e}} \mathrm{XIX} \mathrm{X}^{\mathrm{e}}$ siècle

$10 \mid 2013$

Conte et croyance

\section{La croyance dans les contes de Voltaire}

Faith in Voltaire's Tales

\section{Geneviève Di Rosa}

\section{OpenEdition}

Journals

Édition électronique

URL : http://journals.openedition.org/feeries/900

ISSN : 1957-7753

Éditeur

UGA Éditions/Université Grenoble Alpes

Édition imprimée

Date de publication : 20 septembre 2013

Pagination : 215-232

ISBN : 978-2-84310-253-0

ISSN : 1766-2842

\section{Référence électronique}

Geneviève Di Rosa, «La croyance dans les contes de Voltaire ». Féeries [En ligne], 10 | 2013, mis en ligne le 20 mars 2015, consulté le 08 septembre 2020. URL : http://journals.openedition.org/feeries/ 900

(c) Féeries 


\section{LA CROYANCE DANS LES CONTES DE VOLTAIRE}

ES REPRÉSENTATIONS CULTURELLES des Lumières tendent encore à souligner leur manque de religiosité, leur désintérêt, si ce n'est satirique, pour l'«incroyable besoin de croire», selon le célèbre titre de Julia Kristeva. Pourfendeur inlassable de l'Infâme, Voltaire est la figure emblématique de ce combat de la Raison dénonçant les croyances de la religion chrétienne, assimilées à des superstitions ou à des fictions, jugées de moindre qualité que les fictions mythologiques antiques. Les études récentes des contes voltairiens, tel Le Dialogue philosophique dans les contes de Voltaire d'Annick Azerhad ${ }^{1}$, continuent à mettre l'accent sur l'efficacité satirique du genre et leur volonté systématique de désacralisation de la Bible ainsi que sur leur dénonciation des rituels religieux. Mais elles soulignent également la richesse d'une intertextualité fondée sur la connaissance des exégètes contemporains ainsi que la dimension réflexive du conte : «Écrire un conte devient [...] un acte moins parodique que métalinguistique puisqu'il met en œuvre une réflexion sur le genre, sa portée, ses moyens, sa fonction ${ }^{2}$.» C'est précisément la spécificité des représentations des croyances religieuses dans l'espace littéraire des contes voltairiens que nous proposons d'analyser. Les contes, alors considérés comme genre mineur, ne sont pas un espace neutre ou simplement plaisant de représentation des faits de croyance : qu'apporte ou que déplace l'insertion de ceux-ci dans un espace fictif dévolu au merveilleux?

\section{Les référents socioreligieux des contes philosophiques}

Il est possible de pratiquer une lecture référentielle des contes voltairiens en étudiant les liens tissés avec le contexte socioreligieux propre au XVIII ${ }^{\mathrm{e}}$ siècle.

I. A. Azerhad, Le Dialogue philosophique dans les contes de Voltaire, Paris, Honoré Champion, 2010.

2. P. Cambou, Le Traitement voltairien du conte, Paris, Honoré Champion, 2000, p. 3I. 
Écrits en marge des grands textes de Voltaire portant sur des thématiques religieuses (Le Sermon des Cinquante, Le Dictionnaire philosophique, L'Examen important, La Défense de mon oncle, La Bible enfin expliquée, etc.), les contes reprennent ces thèmes, font allusion aux mêmes événements, aux mêmes controverses, aux mêmes faits religieux qui occupent la scène publique de l'époque. Qu'apporte ce détour par la fiction?

Il nous semble qu'en premier lieu l'espace du conte tend à mettre en relief la discursivité des faits religieux, en les évoquant avant tout comme des actes de langage échangés entre des camps adverses ou comme des matériaux de conversation aptes à être polis par l'usage. L'importance des scènes de repas, de banquet philosophique sur le modèle plutôt dégradé du banquet platonicien, a été déjà soulignée par la critique 3 . Or, les propos afférant au religieux y occupent une place de choix, même dans le cadre mondain fictif des repas parisiens où la politesse exige d'éviter les controverses. À la fin du récit de L'Homme aux quarante écus', le narrateur imagine "un bon souper chez Monsieur André» réunissant entre autres "un docteur de Sorbonne, [...], un capitaine suisse calviniste, deux philosophes et trois dames d'esprit» où "on ne disputa pas plus sur la religion que si personne n'en avait ${ }^{5}$ ", entendre que les sujets attenants au religieux seront abordés sans heurt. En effet, les convives dissertent sur le lien entre la démographie et la foi : la merveilleuse fertilité biblique serait signe d'élection divine pour le docteur de Sorbonne tandis que monsieur André conteste la validité historique des données numériques bibliques en les plaçant sur le même plan que les indications homériques relatives à "Thèbes-aux-cent portes». Il s'agit en fait d'une reprise d'un argumentaire développé dans La Défense de mon oncle 6 participant d'une même entreprise de démystification des récits fabuleux anciens. Le conte met en perspective le débat en le faisant précéder d'une réflexion sur la déperdition démographique induite par l'extension du monachisme : sans l'expliciter, le texte suggère une contradiction au cœur du catholicisme, prônant la fertilité familiale mais détournant nombre de ses fidèles voués à la chasteté. Cependant, ces idées sont bien mieux démontrées, étayées dans les écrits non fictifs. Ici, elles ne sont que reflet, écho partiel, limité, nécessitant pour être resituées

3. Voir C. Mervaud, Voltaire à table, Paris, Desjonquières, 1998; ou A. Azerhad, ouvr. cité.

4. L'Homme aux quarante écus, paru en 1768 chez l'éditeur Cramer à Genève.

5. Dans Romans et contes, éd. R. Pomeau, Paris, GF Flammarion, 1966, p. 439. Il s'agit de notre édition de référence pour toutes les citations des contes de Voltaire.

6. Voir La Défense de mon oncle, chap. IX, «De Thèbes, de Bossuet, et de Rollin». La Défense de mon oncle est lui-même une réplique au Supplément de la Philosophie de l'Histoire de Larcher où l'exemple argumentatif de "Thèbes aux cent portes» est contesté pour son exagération numérique. 
dans leur champ de pensée, de la part du lecteur, une coopération active et des connaissances externes 7 . C'est pourquoi nous pensons plutôt que leur convocation sert à prouver la possibilité d'un langage mondain éclairé par opposition à un langage fanatique : le bon repas de monsieur André est précédé d'une visite d'un homme tout de noir vêtu, «l'œil hagard, les mains fort sales" pour le "conjurer de lui donner à souper avec ses ennemis", ce qui lui sera refusé. Ce "cafard", auteur d'un Antiphilosophique, a certainement pour référent Chaudon ou Paulian, archétypes du fanatisme à l'aune voltairienne ${ }^{8}$. Le narrateur met en scène l'exclusion du scélérat (la section s'intitule "le scélérat chassé») afin de délimiter l'espace de l'interlocution. La scène de repas gagne ainsi en vraisemblance et devient surtout signe d'une communauté discursive adressée au lecteur qui doit adhérer à la croyance en une telle sociabilité. En ce sens, Voltaire est proche de Rousseau traçant une ligne de démarcation entre les interlocuteurs possibles et ceux qui ne parlent pas la même langue', écho d'un temps où des auteurs en mal de reconnaissance tentent de rentrer en relation avec les célèbres philosophes ${ }^{\text {Io }}$. Le conte est le genre de l'anti-pamphlet clérical, lieu d'une pratique langagière mondaine affichée comme manifeste d'un anti-langage fanatique. Les adversaires avec lesquels Voltaire accepte de dialoguer appartiennent à cet espace linguistique commun : le narrateur inclut dans le cercle des convives, le docteur de Sorbonne Pinto, alias abbé Antoine Guénée, auteur des Lettres de quelques juifs à $M$. de Voltaire, que ce dernier appréciait.

Parfois, cependant, la sensibilisation à la discursivité des thématiques religieuses tend à en exhiber l'inanité : les échanges tournent au "pot pourri ", sapant de l'intérieur le sérieux des débats, ou révèlent le caractère logorrhéique des propos déversés. Dans Les Oreilles du comte de Chesterfield,

7. Par exemple, la connaissance de La Défense de mon oncle permet de comprendre la référence à Gargantua dans le conte : il s'agit d'une allusion au chap. 33 où trois conseillers de Picrochole arguent que soumettre Grandgousier, c'est conquérir le monde entier.

8. Dans l'article «fanatisme» du Dictionnaire philosophique, Voltaire écrit : "Guyon, Patouillet, Chaudon, Nonotte, l'ex-jésuite Paulian, ne sont que des fanatiques du coin de la rue, des misérables à qui on ne prend pas garde : mais un jour de Saint-Barthélemy ils feraient de grandes choses.» (Londres, 1764; éd. R. Naves et J. Benda, Paris, Classiques Garnier, 1967, p. 197.)

9. Rappelons la célèbre ouverture de la Lettre à Christophe de Beaumont: «Pourquoi faut-il Monseigneur que j'aie quelque chose à vous dire? Quelle langue commune pouvons-nous parler, comment pouvons-nous nous entendre, et qu'y a-t-il entre vous et moi ?" (Rousseau, Lettre à C. de Beaumont, OC IV, Gallimard, «Bibliothèque de la Pléiade», p. 927.)

IO. D. Masseau dans Les Ennemis des philosophes met en relief cette fascination/répulsion exercée par les philosophes sur leurs adversaires, ces derniers rivalisant d'ouvrages, de libelles dans l'espoir de les faire réagir, de devenir un ennemi digne de joutes écrites: Paris, Albin Michel, 2000. 
le dîner entre «les trois penseurs» est l'occasion de se divertir «à parler de toutes les misères, de toutes les sottises, de toutes les horreurs qui affligent le genre animal ${ }^{\mathrm{II}}$ ", tandis que lors du repas de baptême de l'Ingénu, les convives pris d'ébriété parlent à bâtons rompus :

Les goguenards de Basse-Bretagne dirent qu'il ne fallait pas baptiser son vin ${ }^{12}$. Monsieur le prieur disait que le vin, selon Salomon ${ }^{13}$, réjouit le cœur de l'homme. Monsieur l'évêque ajoutait que le patriarche Juda ${ }^{\mathrm{I} 4}$ devait lier son ânon à la vigne, et tremper son manteau dans le sang du raisin, et qu'il était bien triste qu'on n'en pût faire autant en Basse-Bretagne, à laquelle Dieu a dénié les vignes ${ }^{15}$.

De Salomon au sacrement eucharistique, toutes les références mises dans la bouche des convives, désignés par le terme significatif de "goguenards", exaltent la propension à l'ivresse vinaire. Cet éloge du vin, aux résonances rabelaisiennes, est la caricature grossière d'une lecture allégorique de l'intertexte biblique. Du déversoir incongru aux propos suscitant le conflit, la frontière est ténue et on sait que les conversations des repas des contes sont parfois l'occasion d'affrontements violents relatifs aux croyances, tel par exemple le souper dans Zadig $^{16}$, comme si le langage fanatique bordait en lisière, menaçait d'inversion son anti-modèle.

En second lieu, le détour par la fiction modifie le regard porté sur les faits religieux, il en donne une autre lecture, propre au régime du conte. Le motif de la querelle des refus des sacrements, récurrent tant dans les écrits fictifs que non fictifs de Voltaire, peut être le lieu exemplaire d'observation d'une telle transformation. Rappelons que ce conflit opposant les jansénistes pendant plusieurs décennies à leur hiérarchie ecclésiastique, se focalise autour de l'obligation faite au mourant de montrer un billet de confession produit par un prêtre patenté, autrement dit reconnaissant la bulle Unigenitus. Dans ses écrits historiographiques ${ }^{17}$, Voltaire évoque de façon circonstanciée le contexte de la querelle en pointant le rôle de l'archevêque de Paris, Christophe de Beaumont, tandis que dans ses ouvrages

II. Les Oreilles du comte de Chesterfield (I775), dans Romans et contes, ouvr. cité, chap. V, p. 680.

I2. Allusion à un débat ancien pour établir la nature des oblats requis pour qu'il y ait eucharistie.

13. Allusion certainement aux Proverbes ou bien aux Cantiques des cantiques traditionnellement attribués à Salomon.

I4. Genèse, XLIX : IO-I2.

15. L'Ingénu, chap. IV (1767), dans Romans et contes, ouvr. cité, p. 58-60.

16. Zadig, chap. «Le Souper», 1747: Zadig pense découvrir les charmes d'un repas aux convives cosmopolites mais il lui faudra exercer tous ses talents oratoires pour éviter que le souper ne soit ensanglanté par les querelles religieuses. Dans Romans et contes, ouvr. cité, p. 58-60.

17. Histoire du Parlement de Paris, chap. LXV, 1769 et Précis du Siècle de Louis XV, chap. XXXVI, "Gouvernement intérieur de la France : querelles et aventures depuis I750 jusqu’à I762". 
traitant de thématiques religieuses, il explore les effets humains d'une telle régulation de la mort : dans son Traité sur la tolérance $e^{18}$, le dialogue «entre un mourant et un homme qui se porte bien" marque l'horreur de cette loi au regard de la condition humaine. La périphrase de «l'homme qui se porte bien» désigne un prêtre, appelé «barbare» dans la didascalie des personnages, qui cherche à tout prix à extorquer un billet de confession au malade agonisant. Transposée dans l'espace du conte, l'évocation de la querelle résonne autrement, ainsi qu'on peut le sentir dans le récit d'un Candide tombé malade à Paris :

Un habitué du quartier vint avec douceur lui demander un billet payable au porteur pour l'autre monde : Candide n'en voulut rien faire. Les dévotes l'assurèrent que c'était une nouvelle mode; Candide répondit qu'il n'était point homme à la mode. Martin voulut jeter l'habitué par les fenêtres. Le clerc jura qu'on n'enterrerait point Candide. Martin jura qu'il enterrerait le clerc s'il continuait à les importuner. La querelle s'échauffa : Martin le prit par les épaules, et le chassa rudement; ce qui causa un grand scandale, dont on fit un procès-verbal ${ }^{19}$.

L'écriture travaille à la fois à abstraire le référent de son contexte (la querelle est réduite à un effet de "mode») et à distancier les effets de pathos, la possibilité d'une identification affective grâce notamment à la répétition de parallélismes marquant l'antagonisme de façon mécanique, à force de netteté. Cette distanciation, dans l'énonciation, d'énoncés aux référents religieux, favorise leur transformation paradigmatique : le billet de confession devient le paradigme de l'incongruité, de l'absurdité d'un dogme. Cette dimension est aussi active dans le court récit d'Aventure indienne où le dogme est transposé dans la religion indienne : un Indou est condamné au bûcher "pour avoir soupçonné qu'on pouvait plaire à l'Être suprême par la vertu, sans tenir en mourant une vache par la queue ${ }^{20} »$. Le syncrétisme des dogmes permet de traduire dans la religion indienne l'obligation faite à tout mourant catholique d'un billet de confession tandis que l'argument de la vertu comme salut au regard de Dieu, soutenu par l'Indou condamné, fait penser au positionnement théiste de Rousseau. Mais la substitution analogique est signifiante : la queue de vache est au mourant indien ce que le billet de confession est au mourant français. La sacralité de l'animal réfléchit le sacrement de la confession; en miroir aussi le fait de montrer qu'on tient une queue et le fait de montrer qu'on tient un billet représentent

I8. Dans Traité sur la tolérance, édité en I763 à Genève; rééd. R. Pomeau, Paris, GF Flammarion, I989, chap. XVI.

19. Candide, 1759, dans Romans et contes, ouvr. cité, p. 230.

20. Aventure indienne, I766, dans Romans et contes, ouvr. cité, p. 316. 
la preuve tangible de sa croyance, de l'adhésion au dogme. Le passage par l'analogie propre au conte révèle donc la sémiologie à l'œuvre dans le billet de confession. Que le billet soit demandé, montré, caché, retrouvé, posé sur un meuble, ainsi que le relatent nombre d'anecdotes rapportées par les Nouvelles ecclésiastiques ${ }^{21}$, il s'agit toujours de faire signe de sa croyance.

On voit donc que l'insertion d'un contexte dans l'espace de fiction ne se fait jamais de façon neutre, qu'une forme de praxis s'exerce sur le contexte pour devenir intertexte : le référent externe intégré dans le texte produit par inférences de nouveaux effets de sens.

\section{Le conte, espace de fiction métalinguistique réfléchissant la croyance}

Or, il nous semble significatif que les exemples traités nous mènent à une réflexion sur le langage ou à une réflexion sur les signes de croyance, les deux relevant d'un processus de signification propre à cet espace métalinguistique qu'est le conte pour Voltaire. La fiction permet de repenser la croyance dans sa dimension psychologique - le désir de croire sémiologique - les supports figuratifs de la croyance - , et pragmatique — les effets de la croyance sur le fidèle —, mais aussi de la confronter aux formes de croyance littéraire. Si la croyance religieuse et la croyance fictionnelle relèvent de deux champs épistémologiques distincts, la littérature profane étant au XVIII ${ }^{\mathrm{e}}$ siècle encore perçue comme radicalement autre, néanmoins, de plus en plus de liens sont tissés entre les deux, des problématiques communes se font jour. En effet, nous avons pu observer ${ }^{22}$ qu'une des caractéristiques de la pensée du religieux au XviII ${ }^{\mathrm{e}}$ siècle, tant chez les penseurs orthodoxes qu'hétérodoxes, est de s'intéresser aux questions de la crédulité du sujet, du recours aux supports visibles de croyance, aux miracles, de la distinction in fine entre la foi et la croyance, tandis que les auteurs de fiction s'interrogent sur le degré d'adhésion du lecteur à leur univers, sur la place du merveilleux ou encore sur «la fiction du non-fictif» selon la célèbre expression de Jean Rousset. L'œuvre de Voltaire nous parait emblématique de cette double problématique, notamment par la place accordée à l'intertexte biblique.

2I. L'organe de presse des jansénistes est en effet très friand de tels récits suggérant l'imposture de l'obligation faite au mourant au lieu même du dernier sacrement.

22. Lors de notre thèse de doctorat, Pensée du religieux au siècle des Lumières : analyses sémiostylistiques d'œuvres picturales et littéraires (dir. G. Molinié, U. Paris IV-Sorbonne, nov. 20I2). Thèse à paraître chez Rodopi, collection «Faux titre». 
Avant d'observer l'infléchissement particulier de la réflexion de Voltaire dans deux contes, Le Taureau blanc et L'Ingénu, nous devons préciser quel est le rapport à la Bible au XVIII ${ }^{\mathrm{e}}$ siècle dans le domaine épistémologique. Sous la poussée de la critique morale et historique des saumuriens puis des oratoriens, le mythe biblique chrétien d'un ouvrage d'un seul tenant où le Nouveau Testament est l'accomplissement de l'Ancien Testament est rompu : les abominations vétérotestamentaires sont perçues même chez nombre de catholiques comme irréductibles au champ culturel contemporain; la critique historique tend à diversifier les rédacteurs possibles des livres de la Bible. On observe un consensus tant chez les penseurs orthodoxes qu'hétérodoxes pour reléguer l'Ancien Testament dans le passé, en faire un recueil mémoriel de miracles passés, plus ou moins apprécié, selon qu'on s'inspire ou pas de ce fonds légendaire ${ }^{23}$. Les théologiens versent dans l'apologie aux dépens d'une exégèse évoluant néanmoins vers une approche de plus en plus rationalisante, tandis que les peintres délaissent les sujets narratifs de l'Ancien Testament et que les écrivains évitent le merveilleux chrétien. Les philosophes s'adonnent à la satire et placent au même niveau l'Ancien Testament, Homère et Ovide. Mais, ne nous y trompons pas : ce n'est pas une façon de reconnaître la littérarité de la Bible mais un argument pour dénoncer la mystification chrétienne. Philosophes et penseurs orthodoxes sont dans le même paradigme de pensée de la Bible comme Vérité, les uns pour perpétuer le dogme de la sacralité des Écritures, les autres pour révéler l'imposture, la fabrique à l'œuvre dès le christianisme primitif.

\section{Le Taureau blanc ou la croyance en question}

À première vue, le conte philosophique paraît refléter ce rapport critique, fragmenté et satirique à l'intertexte biblique. Le Taureau blanc, écrit dans la marge de La Bible enfin expliquée ${ }^{24}$, est l'exemple même d'une écriture

23. Ce consensus est notamment remarquable dans l'Encyclopédie de Diderot et d'Alembert où collaborent des théologiens tel Edme Mallet et des penseurs anticléricaux tel Nicolas-Antoine Boulanger, spécialiste de l'étude comparative des mythologies et des cultes. M.-H. Cotoni et B. E. Schwarzbach dans leur article respectif, «Quelques lecteurs de la Bible» et "L’Encyclopédie de Diderot et d'Alembert" (dans Y. Belaval et D. Bourel (dir.), Le Siècle des Lumières et la Bible, éd. Beauchesne, 1986), marquent clairement les limites d'un tel consensus.

24. René Pomeau insiste sur la concomitance des ouvrages dans sa notice de l'édition Romans et contes en GF : «Dom Calmet méritait de figurer au titre de l'œuvre. [...] Pour les Questions sur l'Encyclopédie, qu'il publie depuis 1770, il compulse à nouveau la Bible et Dom Calmet. De ce travail sortira en 1776 La Bible enfin expliquée, commentaire «littéral» selon la méthode de Dom 
composite visant à révéler le merveilleux de l'intertexte biblique vétérotestamentaire. Dans son article consacré à l'analyse narratologique du Taureau blanc, Marie-Hélène Cotoni souligne la mixité des sources :

Cette fantaisie orientale mêle tous les ingrédients d'un conte de fées : amours contrariées d'une jeune princesse, métamorphose d'un prince en bête, animaux qui parlent. Mais ce sont des réminiscences de la Bible qui inspirent le plus souvent Voltaire ${ }^{25}$.

En effet, le narrateur convoque le bestiaire vétérotestamentaire, serpent, ânesse, chien, bouc, corbeau, poisson ainsi que la triade des prophètes David, Jérémie, Ézéchiel, pour littéralement les déplacer, les balader, voire les métamorphoser, en les mêlant à d'autres motifs provenant des mythologies grecque ou égyptienne. L'assimilation des sources mythiques tend à suggérer, mutatis mutandis, que pas plus que les Grecs ne croiraient à leurs mythes ${ }^{26}$, les Français de la cour royale de Louis XV ne croient dans les mythes vétérotestamentaires. Selon M.-H. Cotoni, Voltaire fraie ainsi la voie à une "poésie surréelle", à un espace féérique où à partir du canevas d'un imaginaire ancien d'aventures cocasses, le narrateur ose tisser les liens les plus improbables, les allusions intertextuelles les plus incompatibles. Cependant, l'étude stylistique très fine de M.-H. Cotoni tend à présenter la part féérique du conte comme un heureux supplément, un jeu gratuit mené par un auteur une fois posée «sa volonté de désacralisation», car «ne pas croire à ces histoires n'interdit pas d'y prendre du plaisir ${ }^{27}$ ».

Or, il nous parait pourtant d'autant plus essentiel de réfléchir à l'articulation entre le discours désacralisant et la narration féérique ${ }^{28}$ que le conte est le lieu précisément d'une réflexion méta-textuelle sur ce lien. En effet, le personnage principal de la princesse Amaside exprime son désintérêt violent pour ces récits rabaissés au rang de fables, son incapacité à prendre du plaisir :

Calmet, quoique d'un esprit assez différent. En marge, il a imaginé la facétie du Taureau blanc», "Note sur Le Taureau blanc», ouvr. cité, p. 557.

25. M.-H. Cotoni, "Intertextualité et humour dans Le taureau blanc de Voltaire», Cahiers de Narratologie [En ligne], 2006, mis en ligne le $\mathrm{I}^{\mathrm{er}}$ septembre 2006. URL: $<$ http://narratologie.revues. org/323>.

26. Nous pastichons le célèbre titre de P. Veyne, Les Grecs ont-ils cru à leurs mythes? Essai sur l'imagination constituante, Paris, Seuil, 1983. On sait que Paul Veyne ne répond pas catégoriquement à la question posée puisqu'il s'agit pour lui justement de la déplacer, de la soustraire à la simple alternative du vrai ou du faux afin d'expliciter la complexité d'une réception des mythes allant d'une adhésion naïve au scepticisme critique.

27. M.-H. Cotoni, «Intertextualité et humour dans Le Taureau blanc de Voltaire», art. cité, p. 6.

28. Ce que, d'ailleurs, M.-H. Cotoni fait, partiellement, lorsqu'elle souligne que «la désacralisation entraîne également la suppression de tout symbolisme, au profit de l'absurde, puisque n'est conservée qu'une gestuelle dépourvue de signification ». 
Tous ces contes-là m'ennuient, répondit la Belle Amaside, qui avait de l'esprit et du goût. Ils ne sont bons que pour être commentés chez les Irlandais par ce fou d'Abbadie, ou chez les Velches, par ce phrasier d'Houteville. Les contes qu'on pouvait faire à la quadrisaïeule de la quadrisaïeule de ma grand-mère ne sont plus bons pour moi, qui aie été élevé par le sage Mambrès, et qui ai lu L'Entendement humain du philosophe égyptien nommé Locke, et la Matrone d'Éphèse. Je veux qu'un conte soit fondé sur la vraisemblance, et qu'il ne ressemble pas toujours à un rêve. Je désire qu'il n'y ait rien de trivial ni d'extravagant. Je voudrais surtout que, sous le voile de la fable, il laissât entrevoir aux yeux exercés quelque vérité fine qui échappe au vulgaire. Je suis lasse du soleil et de la lune dont une vieille dispose à son gré, des montagnes qui dansent, des fleuves qui remontent à leur source, et des morts qui ressuscitent; mais surtout quand ces fadaises sont écrites dans un style ampoulé et inintelligible, cela me dégoûte horriblement. Vous sentez qu'une fille qui craint de voir avaler son amant par un gros poisson, et d'avoir elle-même le cou coupé par son propre père, a besoin d'être amusée; mais tâchez de m'amuser selon mon goût ${ }^{29}$.

Amaside raille les tentatives d'adaptation des mythes bibliques à l'esprit moderne par les exégètes orthodoxes, ce qui est un topos de la satire voltairienne. Abbadie et Houteville appréhendent le texte biblique dans une perspective rationaliste, archéologique, tout en se fondant sur la doctrine de l'accomplissement. Le choix de la triade des prophètes peut renvoyer à l'attachement qu'ont ces exégètes aux écrits prophétiques qui permettent la lecture figuriste de l'Ancien Testament par le Nouveau Testament, voire par les événements contemporains. Amaside exprime un dégoût fort pour le répertoire merveilleux et encore plus pour le style biblique, l'hébreu étant alors l'archétype du langage figuratif, dégoût aux accents holbachiens ${ }^{30}$. Cependant, ce qui nous parait troublant dans ce conte est que sa tirade s'insère dans une fiction usant à plusieurs reprises du procédé d'enchâssement de récit dans le récit, de succession de conteurs et de contes, laissant ainsi apparaître en creux la persistance du désir ou du besoin d'adhésion à une fiction. La tentative d'épuisement du fonds légendaire mythique semble ne pas entamer le désir de croyance, dût-il ne se manifester que sous la forme du manque. Le désir de croyance est donné comme besoin de consolation (croire dans une fiction apaiserait les souffrances réelles d'Amaside), ce qui n'est pas sans rappeler la vertu consolatrice de la fréquentation du texte biblique prônée par les théologiens pour

29. Le Taureau blanc, chap. IX : "Comment le serpent ne la consola point », dans Romans et contes, ouvr. cité, p. 583.

30. Le Baron d'Holbach exprime souvent dans ses ouvrages son dégoût fondé sur une doxa des Lumières explicitée notamment par le grammairien Dumarsais pour qui l'hébreu ancien est une «langue stérile», inadaptée à l'esprit moderne et qui appelle de ses vœux la langue de la clarté, émanant de la raison, conceptualisée déjà par Port-Royal. 
qui la consolation est nécessairement liée à une source externe éveillant les affects et la spiritualité, par opposition aux philosophes païens prônant la domination de ses souffrances par la raison et la parole dans l'antique genre rhétorique de la consolatio ${ }^{31}$. Le processus compensatoire psychique qu'est la consolation est mis en œuvre par le personnage du serpent endossant auprès d'Amaside le rôle du conteur consolateur mais il échoue, ce qui est mis en relief par le jeu des titres des chapitres : "Comment le serpent fit des contes pour consoler la princesse» est suivi de "Comment le serpent ne la consola point»! Personnage maître de la persuasion, tentateur mythique par la séduction langagière, le serpent eût dû réussir, si ce n'est que cette fonction entre en contradiction avec une autre : l'éveilleur des consciences, le découvreur de la curiosité humaine, spécifiquement féminine, interprétée par l'Église comme libido sciendi mais présentée par le narrateur comme moteur des lumières naturelles ${ }^{32}$. Ainsi la narration trahit-elle une tension entre croyance et savoir, duperie et découverte, fiction et réalité.

La tirade d'Amaside peut être lue comme un appel à une tentative de conciliation : inventer des fictions qui charmeraient et consoleraient des lecteurs ayant lu Locke. C'est le défi, lancé en vain au serpent, mais peutêtre remporté par le narrateur... Certes, le serpent est un double du narrateur, ce qui est d'autant plus troublant si on songe aux écrits apologétiques contemporains où Voltaire est représenté comme un redoutable serpent ${ }^{33}$. Ce narrateur pratique à profusion le registre merveilleux et s'exerce parfois au style ampoulé pastiche du style biblique, tout ce que déteste Amaside! Mais la réussite du narrateur, sa tentative de réaliser le souhait d'Amaside, tient peut-être alors à la réflexion méta-textuelle, à la mise à distance critique non seulement du merveilleux mais aussi du désir de croire au merveilleux... L'écart entre la pratique jouissive du féérique et le discours anti-féérique est le signe d'un obstacle épistémologique caractéristique de l'écriture voltairienne : signe d'une difficulté à changer de paradigme à

3I. L'être humain ne doit pas chercher en lui-même les consolations qui émanent nécessairement du divin, ou du moins les facultés intérieures doivent être soumises à cette perception sensible du divin.

32. Le narrateur du Taureau blanc évoque en effet à plusieurs reprises le rôle du serpent dans la Genèse, III.

33. Voir N. Brucker, Apologétique, I650-I802 : la nature et la grâce, Recherche en littérature et spiritualité, vol. I6; notamment, p. 293-300, l'article "Censure romaine et apologétique dans la seconde moitié du XVIII ${ }^{e}$ siècle : les enseignements du corpus voltairien " de L. Macé évoquant la métaphore filée par un capucin d'un Voltaire-serpent "qui crache son venin contre le Tribunal de la Sainte Inquisition et contre les missionnaires" (Rome, ACDF, Index, Protocolli 1779-I78I, f. 54.v). 
l'égard de la Bible, à passer de la Bible comme Vérité (ou imposture) à la Bible comme objet culturel, voire littéraire.

\section{L'Ingénu ou l'invention d'un nouveau rapport à l'intertexte biblique}

Cependant, le conte L'Ingénu offre une autre façon de relever ce défi. Le personnage de l'Ingénu est construit autrement que celui d'Amaside : initié à la lecture en même temps qu'au récit biblique, il ne peut éprouver ce sentiment de saturation, de dégoût. Ni lecteur de Locke, ni lecteur des commentaires patristiques ou contemporains, l'Ingénu incarne l'idéal d'un esprit vierge découvrant la Bible. Son apprentissage, véritable parcours initiatique évoqué sur deux chapitres, les III et IV, est corollaire d'une lecture critique, résistante aux discours interprétatifs qu'on veut lui imposer. S'attachant d'abord naïvement puis fanatiquement à la lettre du texte, l'Ingénu veut être baptisé dans un fleuve à l'instar de Jésus dans le Jourdain; découvrant le récit du baptême de l'eunuque, il est confronté à la lecture figuriste de l'Ancien Testament déjà mise en ouvre dans les Actes des apôtres; mais porté par son amour pour Mlle de Saint-Yves, il atteint la révélation de la valeur sacramentelle du baptême. Mû par Éros, le personnage de l'Ingénu s'abreuve à la source vive du texte, non plus littéralement mais par compréhension intime de la langue biblique. Or, ce parcours du personnage réalise dans la fiction le travail de l'écriture à l'égard de l'intertexte biblique qui finit par s'émanciper des transgressives satires des lectures traditionnelles de la Bible. En effet, dans ces deux chapitres, le narrateur ne cesse de tisser des liens avec l'intertexte biblique, d'abreuver son écriture à la source des figures bibliques, mais d'une toute autre façon que la surenchère satirique omniprésente dans l'écriture du Taureau blanc. Pour saisir la particularité de son écriture, nous proposons d'étudier stylistiquement un extrait du chapitre 3 , le moment où les deux personnages féminins, vierges de leur état, désespérés d'attendre en vain l'arrivée du futur baptisé, découvrent en secret l'Ingénu au bain ${ }^{34}$ :

Mademoiselle de Kerkabon pleurait. Mademoiselle de St. Yves ne pleurait pas, mais elle poussait de profonds soupirs qui semblaient témoigner son goût pour les sacrements. Elles se promenaient tristement le long des saules et des roseaux qui bordent la petite rivière de Rance, lorsqu'elles aperçurent au milieu de la rivière une grande figure assez blanche, les deux mains croisées sur la poitrine. Elles jetèrent un grand

34. Nous avons développé plus longuement cette étude stylistique des chap. 3 et 4 de L'Ingénu lors de notre thèse de doctorat (voir supra, n. 22). 
cri et se détournèrent. Mais la curiosité l'emportant bientôt sur toute autre considération, elles se coulèrent doucement entre les roseaux; et quand elles furent bien sûres de n'être point vues, elles voulurent voir de quoi il s'agissait ${ }^{35}$.

La lexie du voyeurisme succède à l'amour rêvé, idéalisé, alors que la lexie du religieux est répartie dans tout le passage. Le jeu est blasphématoire, grâce aux allers-retours d'une lexie à l'autre, et sacrilège grâce à une approche des limites toujours esquissées et jamais atteintes. L'explication toute ironique du narrateur, concernant les "profonds soupirs qui semblaient témoigner son goût pour les sacrements", joue sur la confusion amour sacré / amour profane, secrétée par un dédoublement de la perception plus que de l'énonciation. Le mariage d'une prosodie élégiaque et d'une symbolique phallique joue à plein dans la protase en cadence mineure de la phrase «Elles se promenaient tristement le long des saules et des roseaux qui bordent la petite rivière de Rance...» : l'allitération des sifflantes sonores et sourdes suggère tout autant les peines d'amour que le désir puissant du dieu Pan, se consolant de la mort de la nymphe Syrinx, noyée dans un fleuve, grâce à l'invention de sa flûte de roseaux bruissant ${ }^{36}$. Remarquons également que plusieurs de ces lexies sont des embrayeurs d'une lexie des manifestations corporelles : pleurait, témoigner, se détournèrent, traduisent le langage du corps, et sont donc signes de l'importance accordée à la gestuelle et à l'expressivité.

Cette trame textuelle dans l'économie du conte joue certainement le rôle d'un contrepoint, allégeant la gravité de la scène centrale où a lieu le débat entre l'Ingénu et ceux qui veulent lui imposer un baptême à l'église. Elle peut donc paraître relever d'un vernis de libertinage érotique répandu sur une grande part de la littérature des Lumières ${ }^{37}$. Mais il nous semble qu'elle est plus que cela car non seulement les deux trames se nouent grâce à l'intervention de Mlle de Saint-Yves obtenant la reddition de l'Ingénu mais aussi elles se rejoignent plus en profondeur par le discours ou le dialogue qu'elles entretiennent avec l'intertexte biblique. Ce qui au niveau de l'intrigue narrative se résout, dans l'écriture se résout aussi.

Nous avons vu que le débat opposant l'Ingénu à ses prosélytes se réfere à la lettre biblique à partir d'un texte des Actes des apôtres du Nouveau Testament réfléchissant la lecture typologique chrétienne de l'Ancien

35. L'Ingénu, chap. III : «le Huron, nommé l'Ingénu, converti», ouvr. cité, p. 334.

36. Dans les Métamorphoses d'Ovide, Livre premier.

37. C'est l'interprétation la plus répandue; ainsi R. Barny écrit-il : «le registre grivois apparente L'Ingénu à un conte polisson»; dans Études textuelles, vol. 5, "L'Ingénu : je vous épouse, VI», PU de Franche-Comté, I995, p. 243-264. 
Testament. Or, la courte séquence de l'Ingénu au bain renvoie à un ensemble de récits bibliques qui inter-textuellement travaillent le texte, parfois en tension, l'ouvrent sur un feuilleté de suggestions hypo-textuelles ou plutôt iconographiques car une des particularités du passage est qu'il donne à voir et pas seulement à lire. Ainsi trois représentations visuelles nous viennentelles à l'esprit en lisant la scène : Suzanne au bain du Livre de Daniel ${ }^{38}$, le baptême du Christ dans le Jourdain par Jean-Baptiste ${ }^{39}$, Ève tentatrice dans l'Éden.

Le passage s'apparente en effet à une forme d'inversion parodique du récit biblique de Suzanne au bain : en analyse narratologique, on peut dire que les deux personnages féminins de L'Ingénu occupent la place actantielle du couple de vieillards dans le Livre de Daniel, un même désir de voir l'objet de leur convoitise nu dans son bain les incite à le surprendre. Le rapprochement est d'autant plus justifié et significatif si l'on songe que la célèbre péricope de Suzanne au bain, dont la reconnaissance a été rejetée par les Églises réformées ${ }^{40}$, est intégrée à la liturgie du Carême aux rites prébaptismaux dès les temps patristiques. Dans cette perspective, le couple de Mlle de Kerkabon et Mlle de Saint-Yves pourrait être la transposition des deux servantes accompagnant Suzanne, désirant aider l'Ingénu à l'accomplissement de son bain spirituel. Cependant, bien que la reconnaissance liturgique de la péricope soit effectivement ancrée dans la tradition patristique, au XVIII ${ }^{\text {e }}$ siècle, le choix de la représentation du récit de Suzanne au bain peut poser problème à l'orthodoxie chrétienne. Notamment, dans le domaine de l'iconographie post-tridentine, c'est un sujet religieux à éviter. Il est évident que la storia de Suzanne fournissait aux peintres le prétexte de représenter la nudité et de mettre en scène le voyeurisme, en intégrant dans la composition picturale, l'espace du spectateur. La sécularisation de la thématique allait de pair avec une réflexion esthétique sur la place du spectateur. C'est pourquoi, il nous semble que Voltaire joue à la fois de la symbolique sacramentelle et de l'évolution galante attachées à la péricope, au point de jonction du sacré et du profane.

38. Livre de Daniel, XIII, dans la Bible de Sacy (Paris, Robert Laffont, I990).

39. Épisode synoptique des quatre évangiles : Matthieu III II-I7; Marc I, 7-II; Luc III, I6-22; Jean I, 25-35 (ordre de la Bible de Sacy).

40. Il existe deux versions d'origine du texte grec du Livre de Daniel : l'une provient de la Septante, l'autre de la traduction de l'Ancien Testament hébraïque en grec par Théodotion, juif de culture hellénistique, entre le $\mathrm{I}^{\mathrm{e}}$ et le $\mathrm{II}^{\mathrm{e}}$ siècle ap. J.-C. C'est dans cette dernière seulement qu'on trouve l'histoire de Suzanne : celle-ci est admise comme canonique par l'Église catholique alors que les Églises réformées la refusent. L'attachement de l'Église catholique à ce texte est certainement lié à son interprétation baptismale. 
La référence iconographique au baptême du Christ par Jean-Baptiste est contenue principalement dans la proposition subordonnée «lorsqu'elles aperçurent au milieu de la rivière une grande figure assez blanche, les deux mains croisées sur la poitrine». Il est manifeste qu'il ne s'agit pas d'une réécriture du texte biblique puisqu'aucun des Évangiles synoptiques ne propose ces syntagmes nominaux descriptifs d'un espace, d'une figure et $\mathrm{d}^{\prime}$ une gestuelle ${ }^{4 \mathrm{r}}$. L'insertion de la proposition subordonnée vaut donc plutôt comme ekphrasis d'une des scènes les plus fréquemment représentées dans l'art chrétien et les plus normalisées selon un schéma fixe appelant peu de variantes. Le syntagme "la grande figure assez blanche» connote la mise en valeur dans l'espace de la toile de la figure christique, représentée au premier plan et au centre, donnée comme source de lumière émanant de la blancheur de sa chair peinte; la perception de la centralisation de la figure est renforcée par la locution adverbiale «au milieu de». La posture du corps est exprimée par le syntagme «les deux mains croisées sur la poitrine» : cette représentation gestuelle est on ne peut plus classique dans l'art chrétien (avec la variante des mains jointes) et fait clairement de l'Ingénu une représentation christomorphe. Comment interpréter cette assimilation, via les suggestions picturales, du Huron au Christ? Pour les chrétiens, la scène du baptême est devenue le lieu de réalisation du parcours christique dans sa totalité : l'immersion, où se morfond l'Ingénu, est le temps de la souffrance et de la mort, tandis que l'émersion correspond à la fin de la purification qui permet la Résurrection. L'assimilation est-elle un pur blasphème de la part d'un Voltaire iconoclaste? La visée transgressive jubilatoire ne fait pas de doute, comme nous avons déjà pu l'observer, mais nous pensons que le narrateur, tout en jouant avec ces connotations, ces allusions, suggère ici le possible martyre d'une jeunesse sacrifiée aux errances des adultes : on sait que le patriarche de Ferney a été personnellement choqué par la mort du jeune chevalier de la Barre et qu'il s'est beaucoup investi dans sa défense ou sa réhabilitation ${ }^{42}$; or, c'est dans la même période qu'il invente le personnage de l'Ingénu qui peut être vu comme l'emblème d'une jeunesse porteuse de régénération mais en même temps menacée. L'intrépide Huron échoue à la Bastille, comme un certain Voltaire du temps de sa jeunesse. La tension du texte est aussi là dans cet affleurement

4I. D’ailleurs, les Évangiles n'en donnent aucun, se contentant de mentionner l'événement et tout au plus de désigner le lieu : "Jésus ayant été baptisé sortit aussitôt hors de l'eau» (Matthieu); "Jésus fut baptisé par Jean dans le Jourdain" (Marc); "Jésus ayant été aussi baptisé» (Luc).

42. Il rédige en 1767 la Relation de la mort du chevalier de La Barre à Monsieur le marquis de Beccaria et Le Cri d'un sang innocent. 
latent sous le jeu, l'humour, la satire, d'une violence toujours possible entre hommes. Ce qui est d'ailleurs une compréhension intime du christianisme, religion non pas du Livre mais du Christ bafoué, violenté, martyrisé, tel qu'il aurait été prophétisé dans le Livre d'Isaïe...

La troisième référence biblique visuelle appert essentiellement dans la dernière phrase, en particulier dans la proposition "elles se coulèrent doucement entre les roseaux». Le verbe pronominal se couler s'est forgé par dérivation métaphorique et conserve encore une puissante résonance connotative ici activée par le contexte. Les sèmes de liquidité et de flexibilité surgissent par association avec la double occurrence de rivière et de roseaux. La représentation figurée d'une métamorphose aqueuse ou animale des deux demoiselles, mues par une curiosité irrépressible, incite à l'association idéelle avec le serpent édénique. Tentées et tentatrices, proies et prédatrices, elles sont les nouvelles Ėve peccamineuses, régénérées par le baptême. La labilité même de leurs fonctions actantielles fait des deux personnages féminins le symbole de la réversibilité de la nature humaine. Voltaire a parfaitement saisi que l'intérêt du symbole est d'être opérateur d'inversion. Dans le feuilleté des représentations poly-symboliques, les différentes strates se font écho, s'opposent ou s'harmonisent tel le rapprochement entre Ève et les servantes de Suzanne ou Ève et les vieillards libidineux ou encore la contamination des deux scènes bibliques de Suzanne au bain et du baptême du Christ.

Ces différentes sollicitations d'images bibliques, suggestions visuelles à partir du grain levant des mots, dans la pensée du lecteur, ne sont pas coupées du travail scripturaire dans la mesure où, comme nous avons pu l'observer, les lexies des manifestations corporelles et de la vision informent essentiellement le récit. Le texte réalise ce qu'il énonce, les lexies sont en correspondance avec les effets du texte : parler de vision en donnant en même temps à voir, autrement dit mettre en scène le désir de voir et le réaliser par l'écriture en suscitant des représentations visuelles chez le lecteur. On comprend mieux alors pourquoi Voltaire a choisi le sacrement du baptême comme motif narratif de l'éducation biblique de l'Ingénu. En effet, dans le Dictionnaire philosophique, à l'article "Baptême», l'énonciateur souligne le lien entre ce sacrement et les manifestations sensibles : "Baptême, mot grec qui signifie immersion. Les hommes qui se conduisent toujours par les sens, imaginèrent aisément que ce qui lavait le corps lavait aussi l'âme ${ }^{43}$.» Les femmes, qui se conduisent aussi par les sens, trouvent

43. Dictionnaire philosophique, article "Baptême», ouvr. cité, p. 46. 
à parfaire leur plaisir sensible par la contemplation du baptisé, tandis que le lecteur fouette sa capacité d'imagination à partir d'images et de lexies. La trame textuelle des amours des mesdemoiselles ne se limite donc pas à tracer la veine du libertinage érotique et blasphématoire, elle permet de sortir de l'aporie mise en scène entre une approche de la religion basée sur des usages évidés de sens et une lecture littérale de la Bible attachée fanatiquement à la lettre, en mettant en ouvre un autre rapport au texte biblique, grâce à un dialogue fructueux et très libre. Ce travail, subtil, de suggestions visuelles et hypo-textuelles dans le feuilleté des mots, manifeste la créativité de Voltaire mais aussi la richesse des images de la Bible et des siècles de culture chrétienne : la puissance imageante de l'écriture voltairienne vient s'aboucher à celle de la Bible. Mais elle permet aussi de mettre en relief l'idée que la croyance passe par des rituels, une économie des signes où la signification retrouve sa place.

À l'épuisement du réservoir légendaire, à la violence faite au texte biblique, à l'évidement de toute symbolique par crainte de l'allégorie caractéristique du Taureau blanc s'opposent donc une nouvelle façon de dialoguer avec la Bible, une prise en considération de sa richesse figurative et polysémique, une réflexion, déliée, sur les signes rituels, les supports symboliques de la croyance. L'attention portée au grain du texte permet de ne pas nous arrêter aux positionnements idéologiques connus de Voltaire et nous incite à émettre l'hypothèse que l'espace littéraire du conte a pu lui permettre de dépasser l'obstacle épistémologique d'une Bible appréhendée sous l'angle de sa vérité ou de son imposture pour aller vers un travail de réécriture littéraire d'un texte perçu dans sa dimension culturelle. La fiction est le lieu de réalisation des impensés de son auteur ou de ce qui pouvait difficilement se penser dans le cadre de ses écrits plus théoriques.

\section{Réécrire pour penser...}

Que l'intertexte biblique soit perçu comme un réservoir figé de formes, de thèmes et de propos où l'on puise ad libitum — pratique de maltraitance de l'intertexte biblique dont use souvent Voltaire — ou que s'instaure une réécriture poétique vivifiante, sa présence marque la nécessité de donner forme à l'invisible, de médiatiser le désir de croyance. Dans Pot pourri, l'énonciateur apostrophe Rousseau en fuite pour qu'il lui explique le théisme :

- Mais, monsieur Jean-Jacques, comment vous êtes-vous fait théiste? quelle cérémonie faut-il pour cela? - Aucune, nous dit Jean-Jacques. Je suis né protestant, j'ai retranché tout ce que les protestants condamnent dans la religion romaine. Ensuite, 
j'ai retranché tout ce que les autres religions condamnent dans le protestantisme : il ne m'est resté que Dieu ${ }^{44}$.

L'ironie affleure dans les questions de l'énonciateur à l'égard du concept d'une religion sans signe, sans rituel, ce qui n'est pas sans nous rappeler la façon dont Voltaire dans Aventure indienne place face à face le dogme imposant de tenir la queue d'une vache en mourant et une religion fondée uniquement sur l'idéal de vertu ${ }^{45}$. De même, dans le conte Memnon, le narrateur trahit son impuissance à représenter un "esprit céleste» apparu en songe sans recourir aux figurations connues : «il avait six belles ailes mais ni pieds, ni tête, ni queue, et ne ressemblait à rien ${ }^{46} "$ !

La réflexion sur la croyance marque la capacité de Voltaire à percevoir la valeur anthropologique des croyances pratiquées. Il s'agit là encore d'une vraie difficulté épistémologique pour un penseur du XvIII ${ }^{\mathrm{e}}$ siècle d'accepter de reconnaître que les mythes contiennent un savoir sur les hommes mais tout se passe comme si la transposition du surnaturel dans l'espace fictif du conte avait permis à Voltaire d'appréhender la sagesse contenue dans les mythes, de dépasser la dénonciation d'une imposture pour saisir ce qu'ils nous disent de l'homme. La réflexion anthropologique émane notamment du choix qui est fait des représentations narratives de certaines croyances. Plusieurs commentateurs ont remarqué dans nombre des contes voltairiens une figuration des repas, nourriture et boisson, qui traduit le lien des pratiques alimentaires avec la culture et les mythes : "Voltaire pressent l'importance de ces phénomènes, même s'il les traite par l'ironie ${ }^{47}$." Les contes présentent de façon récurrente le christianisme comme religion organisée autour du culte du vin, ce qui est une représentation inquiète de la croyance : croire serait perdre la raison, au profit des sens et de la déraison... En même temps, le conte Le Crocheteur borgne suggère que la déraison fait partie de la raison, l'ivresse transporte dans un lieu de délices Mesrour le crocheteur borgne, alors que l'eau sacrée des ablutions propres aux rites musulmans qu'il reçoit par mégarde lui fait l'effet d'une douche froide le réveillant brutalement de son songe ${ }^{48}$ ! Dans les Lettres d'Amabed,

44. Pot-pourri, 1765, dans Romans et contes, ouvr. cité, p. 299.

45. Voir supra, p. 4.

46. Memnon, dans Romans et contes, ouvr. cité, p. II4.

47. C. Merveaud, Voltaire à table, ouvr. cité, p. I7o.

48. Le Crocheteur borgne: Mesrour qui se livre à l'échauffement de son imagination par l'alcool trouve le bonheur dans les songes à l'opposé de Memnon, autre personnage borgne des contes, qui pense que la sagesse consiste dans l'abstinence et sombre dans une succession de malheurs. Dans Romans et contes, ouvr. cité, p. 597. 
le parcours à rebours d'un roman d'apprentissage des musulmans ne vient pas du fait que captifs, ils absorbent force vin et cochonnailles, mais qu'ils abandonnent toute régulation de leurs appétits. Les croyances apparaissent à la fois comme force de régulation et de dérèglement. Les contes ouvrent la perspective d'une catastrophe ontologique de l'être humain dont on peut suivre la trace à travers la cohorte de figures fictives de martyrs, d'êtres violentés, mutilés dans leur chair. 